\title{
Erratum
}

\section{Erratum: Hauben et al., "Passive or Active Immunization with Myelin Basic Protein Promotes Recovery from Spinal Cord Injury Contusion"}

In the article "Passive or Active Immunization with Myelin Basic Protein Promotes Recovery from Spinal Cord Injury Contusion" by Ehud Hauben, Oleg Butovsky, Uri Nevo, Eti Yoles, Gila Moalem, Eugenia Agranov, Felix Mor, Raya Leibowitz-Amit, Evgenie Pevsner, Solange Akselrod, Michal Neeman, Irun R. Cohen, and Michal Schwartz, which appeared on pages 6421-6430 of the September 1, 2000 issue, the following error was discovered:

In the legend of Figure 4A, a reference is missing. Hauben E, Nevo U, Yoles E, Moalem G, Agranov E, Mor F, Akselrod S, Neeman M, Cohen IR, Schwartz M (2000) Autoimmune T cells as potential neuroprotective therapy for spinal cord injury. Lancet 355:28-287, which is cited in the text of the article, should be cited in the legend as well. The sentence in the legend that reads "For comparison, a similar experiment using five PBS-treated and six rats treated immediately with anti-MBP T cells is shown here" should be replaced by "For comparison, a similar experiment, taken from Hauben et al. (2000), of six rats treated immediately with anti-MBP is shown."

DOI: 10.1523/JNEUROSCI.0251-16.2016 